\title{
State Estimation Using Quantized Measurements
}

\author{
Minyue Fu* and Carlos E. de Souza** \\ * School of EECS, University of Newcastle, Callaghan, NSW 2308 \\ Australia (email: minyue.fu@newcastle.edu.au) \\ ** Department of Systems and Control, Lab. Nacional de Computação \\ Cientifica, Petrópolis, RJ 25651-075, Brazil (email: csouza@lncc.br)
}

\begin{abstract}
In this paper we consider the problem of state estimation for linear dynamic systems using quantized measurements. This problem arises when state estimation needs to be done using information transmitted over a digital communication channel. We investigate how to design the quantizer and the estimator jointly.
\end{abstract}

\section{INTRODUCTION}

In this paper, we study the problem of state estimation using quantized measurements transmitted over a digital communication channel. The use of such a channel limits the amount of information able to be transmitted. Therefore, it is desirable to know how to quantize the measured signal so that good state estimation can be achieved using limited information. A lot of research has happened in the last decade or so for feedback control problems using quantized measurements; see, for example, Wong and Brockett (1999), Baillieul (2001), Brockett and Liberzon (2000), Elia and Mitter (2000,2001), Nair and Evans (2003), Tatikonda and Mitter (2004), and Fu and Xie $(2005,2006)$.

Traditional quantizers employ linear quantization, i.e., the quantization grids are equally spaced. While this type of quantizers tends to preserve information well when the input signal falls into the dynamic range of the quantizer, the number of quantization levels required for a given quantization step-size increases linearly as the dynamic range increases. This paper considers logarithmic quantizers where the quantization step-size grows exponentially as the input increases. The use of logarithmic quantizers is motivated by the fact they are shown to outperform linear quantizers in control problems, as demonstrated by Elia and Mitter $(2000,2001)$, and Fu and Xie $(2005,2006)$. We show in this paper that logarithmic quantizers also work well for state estimation problems.

\section{PROBLEM FORMULATION}

Consider the following linear system:

$$
\begin{aligned}
x(k+1) & =A x(k)+B w(k), \quad x(0)=x_{0} \\
y(k) & =C x(k)+v(k)
\end{aligned}
$$

where $x(k) \in \mathbf{R}^{n}$ is the state, $w(k) \in \mathbf{R}^{m}$ is the process noise, $y(k) \in \mathbf{R}$ is the measurement, $v(k) \in \mathbf{R}$ is the measurement noise, and $A, B$ and $C$ are known matrices of appropriate dimensions. It is assumed that $x_{0} \in \mathbf{R}^{n}$ is a random variable with mean $\bar{x}_{0}$ and covariance matrix $\Sigma_{0}$, and $w$ and $v$ are uncorrelated zero-mean white noises with covariances $\Sigma_{w}$ and $\Sigma_{v}$, respectively, and they are uncorrelated with $x_{0}$.

The quantized estimator we consider consists of three parts: a quantizer, a digital communication channel and an estimator, as shown in Fig. 1. Instead of quantizing the measured signal directly, we choose to quantize the prediction error of the estimator:

$$
\begin{aligned}
\hat{x}(k+1) & =A \hat{x}(k)+L Q(y(k)-\hat{y}(k)), \quad \hat{x}(0)=\bar{x}_{0} \\
\hat{y}(k) & =C \hat{x}(k)
\end{aligned}
$$

where $\hat{x}(k) \in \mathbf{R}^{n}$ and $\hat{y}(k) \in \mathbf{R}$ are estimates of $x(k)$ and $y(k)$, respectively, $Q(\cdot)$ is the quantizer, and $L$ is the estimator gain. The quantity

$$
\varepsilon(k)=y(k)-\hat{y}(k)
$$

is the prediction error, and the quantization error will be denoted by $\varepsilon_{q}(k)$, i.e.,

$$
\varepsilon_{q}(k)=\varepsilon(k)-Q(\varepsilon(k)) .
$$

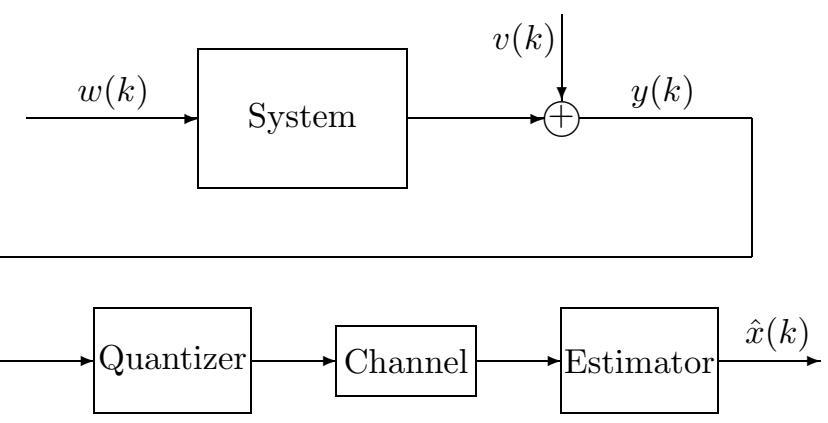

Fig. 1. Quantized state estimation.

Note that state estimation is constructed only using the quantized prediction error. Hence, under the ideal channel assumption, both sides of the channel can construct the same estimate using the quantized prediction error.

This paper considers static quantizers. A static quantizer takes one input sample and produces one output sample without referring back to the previous input samples. Our objective of quantized state estimation is similar to that of stationary Kalman filter (see, Anderson and Moore, 1979), 
namely we want to minimize the asymptotic variance of the estimation error defined by:

$$
\lim _{k \rightarrow \infty} \mathcal{E}\left\{(x(k)-\hat{x}(k))^{T}(x(k)-\hat{x}(k))\right\}
$$

subject to certain constraints on the quantizer, where in the above $\mathcal{E}\{\cdot\}$ denotes mathematical expectation.

\section{STATE ESTIMATION WITH LOGARITHMIC QUANTIZATION}

A logarithmic quantizer has quantization levels given by

$$
\mathcal{V}=\left\{\mu_{i}=\rho^{i} \mu_{0}: i=0, \pm 1, \pm 2, \cdots\right\} \cup\{0\}, \mu_{0}>0
$$

where $\rho \in(0,1)$ represents the quantization density. A small $\rho$ implies coarse quantization, whereas a large $\rho$ means dense quantization. The associated quantizer $Q(\cdot)$ is depicted in Fig. 2 and is defined as follows:

$$
Q(\varepsilon)= \begin{cases}\rho^{i} \mu_{0}, & \text { if } \frac{1}{1+\delta} \rho^{i} \mu_{0}<\varepsilon \leq \frac{1}{1-\delta} \rho^{i} \mu_{0} ; \\ 0, & \text { if } \varepsilon=0 \\ -Q(-\varepsilon), & \text { if } \varepsilon<0\end{cases}
$$

where

$$
\delta=(1-\rho) /(1+\rho)
$$

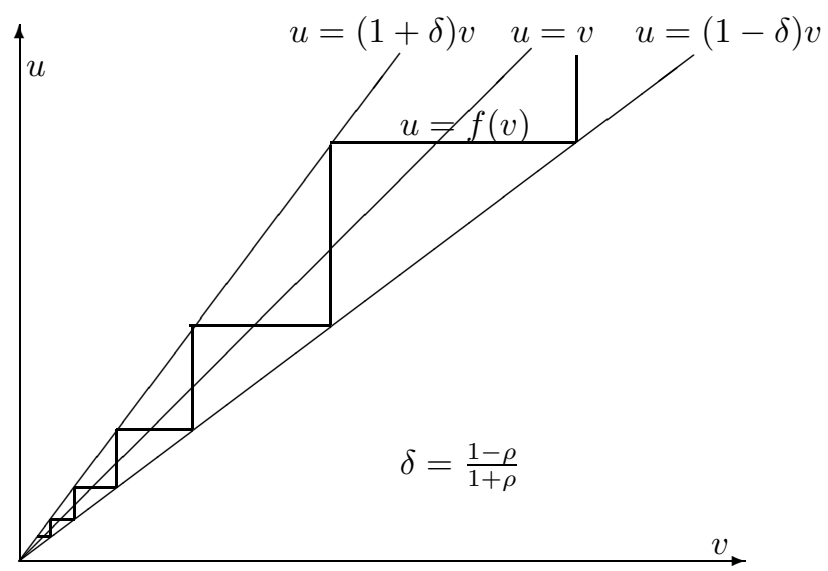

Fig. 2. Logarithmic quantizer.

\subsection{Basic Properties}

Defining the estimation error $e(k)=x(k)-\hat{x}(k)$, the estimation error dynamics can be described as follows:

$$
\begin{aligned}
e(k+1) & =A e(k)+B w(k)-L Q(\varepsilon(k)) \\
\varepsilon(k) & =C e(k)+v(k)
\end{aligned}
$$

Our task is to choose $\rho$ and $L$ so that the asymptotic estimation error variance in (5) is minimized.

As observed in Fu and Xie (2005), a logarithmic quantizer is easily bounded by a sector bound, namely

$$
|Q(\varepsilon)-\varepsilon| \leq \delta|\varepsilon| \text {. }
$$

Using the above, we may rewrite (9) as

$$
e(k+1)=A e(k)-L \varepsilon(k)+B w(k)+L \Delta(k) \varepsilon(k)
$$

where

$$
\Delta(k)= \begin{cases}\varepsilon_{q}(k) / \varepsilon(k), & \text { if } \varepsilon(k) \neq 0 \\ 0, & \text { otherwise }\end{cases}
$$

with the property that $|\Delta(k)| \leq \delta$ for all $k$.

Given the sector bound for $\Delta(k)$ as above, we consider an auxiliary uncertain system defined by

$$
\begin{aligned}
z(k+1)= & (A-L C) z(k)-L v(k)+B w(k) \\
& +L \Delta_{k}[C z(k)+v(k)], \quad\left|\Delta_{k}\right| \leq \delta
\end{aligned}
$$

Note that (13) differs from (11) in the sense that $\Delta_{k}$ is an arbitrary function, whereas $\Delta(k)$ in (11) is due to the quantizer $Q(\cdot)$. It turns out that $\Delta(k)$ in $(11)$ can be viewed as a special instance of $\Delta_{k}$.

Some basic features for the auxiliary system are in order:

Theorem 1. The estimation error dynamics (11) has the following properties:

(1) If the probability density functions of $x(0)-\bar{x}_{0}, w(k)$ and $v(k)$ are even, then the estimation error $e(k)$ has zero-mean and its probability density function is even for all $k \geq 0$;

(2) The estimation error dynamics (9) is quadratically stable if and only if the auxiliary system (13) is quadratically stable, i.e., there exists a matrix $X=$ $X^{T}>0$ such that

$$
e^{T} X e>(A e-L Q(C e))^{T} X(A e-L Q(C e))
$$

for all nonzero $e \in \mathbf{R}^{n}$ if and only if there exists a matrix $P=P^{T}>0$ such that

$$
P>(A-L(1+\Delta) C)^{T} P(A-L(1+\Delta) C)
$$

for all $|\Delta| \leq \delta$

(3) If the system (13) is quadratically stable, then the covariance matrix of $e(k)$ is bounded;

(4) The minimum quantization density $\rho_{\text {inf }}(L)$ for the auxiliary system above to be quadratically stable for a given $L$ is given by

$$
\rho_{\text {inf }}(L)=\left(1-\delta_{\text {sup }}(L)\right) /\left(1+\delta_{\text {sup }}(L)\right)
$$

where

$$
\delta_{\text {sup }}(L)=1 /\left\|C(z I-A+L C)^{-1} L\right\|_{\infty} .
$$

Proof: The first property can be easily shown by induction. Since $\hat{x}(0)=\bar{x}_{0}$, then $e(0)$ is zero-mean with an even probability density function. Note that $Q(\cdot)$ is an odd function. Suppose $e(k)$ is so too for some $k$. Then, it follows from (11) that $e(k+1)$ is also zero-mean with an even probability density. Hence, by induction, $e(k)$ is zero-mean with an even probability density function for all $k \geq 0$.

The second property is proved in Fu and Xie (2005).

To show the third property, we assume that (15) holds for some matrix $P=P^{T}>0$. Since (15) is a strict inequality, it follows that

$$
(1-2 \eta) P>(A-L(1+\Delta) C)^{T} P(A-L(1+\Delta) C)
$$

for some sufficiently small scalar $\eta>0$. Next, define the Lyapunov function candidate $V(e)=e^{T} P e$ for system (11). Considering (11) it follows that

$$
\begin{array}{r}
V(e(k+1))=[A e(k)-L(1+\Delta(k)) \varepsilon(k)+B w(k)]^{T} P \\
\cdot[A e(k)-L(1+\Delta(k)) \varepsilon(k)+B w(k)]
\end{array}
$$




$$
\begin{gathered}
=e^{T}(k)[A-L(1+\Delta(k)) C]^{T} P[A-L(1+\Delta(k)) C] e(k) \\
+[L(1+\Delta(k)) v(k)+B w(k)]^{T} P \\
\cdot[L(1+\Delta(k)) v(k)+B w(k)] \\
-e^{T}(k)[A-L(1+\Delta(k)) C]^{T} P \\
\cdot[L(1+\Delta(k)) v(k)+B w(k)] \\
-[L(1+\Delta(k)) v(k)+B w(k)]^{T} P \\
\cdot[A-L(1+\Delta(k)) C] e(k) \\
\leq(1+\tau) e^{T}(k)[A-L(1+\Delta(k)) C]^{T} P \\
\cdot[A-L(1+\Delta(k)) C] e(k) \\
+(1+1 / \tau)[L(1+\Delta(k)) v(k)+B w(k)]^{T} P \\
\cdot[L(1+\Delta(k)) v(k)+B w(k)]
\end{gathered}
$$

for any $\tau>0$. Choosing $1+\tau=(1-\eta) /(1-2 \eta)$ implies:

$V(e(k+1)) \leq(1-\eta) V(e(k))+m_{1} v^{2}(k)+m_{2} w^{T}(k) w(k)$

for some sufficiently large scalars $m_{1}$ and $m_{2}$ independent of $k$. Applying the result above recursively, we obtain

$$
\begin{aligned}
V(e(k)) \leq & (1-\eta)^{k} V(e(0)) \\
& +\sum_{i=1}^{k}(1-\eta)^{k-i}\left[m_{1} v^{2}(i)+m_{2} w^{T}(i) w(i)\right] .
\end{aligned}
$$

Taking mathematical expectation and denoting by $E(k)$ the covariance matrix of $e(k)$, the latter inequality implies:

$$
\begin{aligned}
\operatorname{Tr}(E(k)) \leq & \frac{1}{\lambda_{\min }(P)}\left[(1-\eta)^{k} \mathcal{E}\{V(e(0))\}\right. \\
& \left.+\left(m_{1} \Sigma_{v}+m_{2} \operatorname{Tr}\left(\Sigma_{w}\right)\right) \sum_{i=1}^{k}(1-\eta)^{k-i}\right] \\
\leq & \tilde{m}_{0} \operatorname{Tr}(E(0))+\tilde{m}_{1} \Sigma_{v}+\tilde{m}_{2} \operatorname{Tr}\left(\Sigma_{w}\right)
\end{aligned}
$$

for some constants $\tilde{m}_{0}, \tilde{m}_{1}$ and $\tilde{m}_{2}$, where $\operatorname{Tr}(\cdot)$ denotes matrix trace. Hence, $E(k)$ is bounded.

The fourth property follows from the known fact that system (13) is quadratically stable if and only if the $H_{\infty}$ norm of $C(z I-A-L C)^{-1} L$ is less than $1 / \delta$; see Packard and Doyle (1990). Therefore, the largest $\delta$ to maintain quadratic stability is given by (17) and the minimum quantization density $\rho_{\text {inf }}(L)$ is related to $\delta_{\text {sup }}(L)$ by (16).

\subsection{Asymptotic Covariance of Estimation Error}

We now proceed to quantify the asymptotic covariance of $e(k)$. Denote by $E(k)$ the covariance matrix of $e(k)$ and its asymptotic version by $E=\lim _{k \rightarrow \infty} E(k)$. We assume that $\rho>\rho_{\text {inf }}(L)$ so that $E(k)$ is bounded (by Theorem 1). It is also assumed that $w(k)$ and $v(k)$ are Gaussian distributed. Moreover, we will denote by $\sigma_{\varepsilon}^{2}$ and $\sigma_{q}^{2}$ the asymptotic variance of $\varepsilon(k)$ and $\varepsilon_{q}(k)$, respectively, and define

$$
\tilde{\delta}^{2}=\sigma_{q}^{2} / \sigma_{\varepsilon}^{2}
$$

to be the normalized quantization error variance.

The computation of $E(k)$ is complicated by the fact that $Q(\cdot)$ is a nonlinear function. But when the number of quantization levels is not too small, the following conditions hold very well in numerical simulations.

C1. The quantization error $\varepsilon_{q}(k)$ is uncorrelated with $\tilde{e}(k+1)=A e(k)-L \varepsilon(k)+B w(k)$ (note that the latter is the predicted state estimation error without quantization error);

C2. The prediction error $\varepsilon(k)$ is approximately Gaussian distributed.

Under Condition $\mathrm{C} 2$, we may relate the variance of the quantization error $\varepsilon_{q}(k)$ to that of the prediction error $\varepsilon(k)$. We observe that $\varepsilon_{q}(k)$ is influenced by the choice of $\mu_{0}$ in (7). However, two simple properties are easily observed from (7):

P1. $\varepsilon_{q}(k)$ is periodic in $\mu_{0}$ in a logarithmic scale, i.e., if $\mu_{0}$ is multiplied by $\rho^{j}$ for any integer $j, \varepsilon_{q}(k)$ remains the same;

P2. A logarithmic quantizer is linearly scalable in the sense that if $\varepsilon(k)$ is multiplied by $\rho^{j}$ for any integer $j$, then $\varepsilon_{q}(k)$ is multiplied by the same factor.

In fact, it turns out that the influence of $\mu_{0}$ on $\varepsilon_{q}(k)$ is negligible for small values of $\delta$. This means that $\sigma_{q}^{2}$ is approximately proportional to $\sigma_{\varepsilon}^{2}$ for a given $\delta$.

For a Gaussian distributed prediction error, the assertion above can be well justified by numerical simulations and the actual $\tilde{\delta}^{2}$ can be well approximated by

$$
\tilde{\delta}^{2} \approx\left(1+0.45 \delta^{2}\right) \delta^{2} / 3 .
$$

We now estimate the asymptotic covariance matrix of $e(k)$.

Theorem 2. Consider the quantized estimation error dynamics (9). Suppose Conditions C1 and C2 and the approximation (19) for $\tilde{\delta}$ hold. Denote by $\tilde{E}(k)$ an approximation of $E(k)$. Then $E(k+1)$ can be approximated by

$$
\begin{gathered}
\tilde{E}(k+1)=(A-L C) \tilde{E}(k)(A-L C)^{T}+\tilde{\delta}^{2} L C \tilde{E}(k) C^{T} L^{T} \\
+\left(1+\tilde{\delta}^{2}\right) L \Sigma_{v} L^{T}+B \Sigma_{w} B^{T} .
\end{gathered}
$$

Moreover, if system (9) is quadratically stable, then $\tilde{E}=$ $\lim _{k \rightarrow \infty} \tilde{E}(k)$ generated by (20) exists and is finite. It follows that the asymptotic covariance matrix $E$ can be approximated by the solution $\tilde{E}=\tilde{E}^{T} \geq 0$ to the following generalized discrete-time Lyapunov equation:

$$
\begin{gathered}
\tilde{E}=(A-L C) \tilde{E}(A-L C)^{T}+\tilde{\delta}^{2} L C \tilde{E} C^{T} L^{T} \\
+\left(1+\tilde{\delta}^{2}\right) L \Sigma_{v} L^{T}+B \Sigma_{w} B^{T} .
\end{gathered}
$$

Proof: Using Condition $\mathrm{C} 1$ and the properties of $w(k)$ and $v(k)$, it is straightforward to see that

$$
\begin{gathered}
E(k+1)=(A-L C) E(k)(A-L C)^{T}+B \Sigma_{w} B^{T} \\
+L \Sigma_{v} L^{T}+L \mathcal{E}\left\{\varepsilon_{q}^{2}(k)\right\} L^{T} .
\end{gathered}
$$

Then, we can get (20) by using (18) and considering that

$$
\mathcal{E}\left\{\varepsilon^{2}(k)\right\}=C E(k) C^{T}+\Sigma_{v} .
$$

Now suppose (9) is quadratically stable. By Theorem 1 , $\left\|\delta C(z I-A+L C)^{-1} L\right\|_{\infty}<1$. Using the discrete-time bounded-real lemma (de Souza and Xie, 1992), there exists a matrix $\Omega=\Omega^{T}>0$ such that 


$$
\begin{aligned}
& 1-\delta^{2} C \Omega C^{T}>0, \\
& \Omega-L L^{T}-A_{e}\left(\Omega^{-1}-\delta^{2} C^{T} C\right)^{-1} A_{e}^{T}>0
\end{aligned}
$$

where $A_{e}=A-L C$. Since $\left(\Omega^{-1}-\delta^{2} C^{T} C\right)^{-1} \geq \Omega$, the above inequalities imply

$$
\Omega-A_{e} \Omega A_{e}^{T}>\delta^{2} L C \Omega C^{T} L^{T} .
$$

We denote

$$
\bar{E}=\alpha \Omega, \quad W=\left(1+\tilde{\delta}^{2}\right) L \Sigma_{v} L^{T}+B \Sigma_{w} B^{T}
$$

where $\alpha>0$ is a scaling parameter. Since (22) is linear in $\Omega$ and is a strict inequality, it follows that there exists a sufficiently large $\alpha>0$ such that $\bar{E} \geq \Sigma_{0}$ and

$$
\bar{E}-A_{e} \bar{E} A_{e}^{T}>\delta^{2} L C \bar{E} C^{T} L^{T}+W .
$$

We will show that $\bar{E} \geq \tilde{E}(k)$ for all $k \geq 0$. This can be proved by induction. Note that $\bar{E} \geq \Sigma_{0}=\tilde{E}(0)$ and $\tilde{\delta} \leq \delta$. Suppose $\tilde{E}(k) \leq \bar{E}$ for some $k$. Then, from (20) and (23),

$$
\tilde{E}(k+1) \leq A_{e} \bar{E} A_{e}^{T}+\delta^{2} L C \bar{E} C^{T} L^{T}+W \leq \bar{E} .
$$

Hence, $\bar{E}$ is indeed an upper-bound of $\tilde{E}(k)$ for all $k$.

Now we use the result above to show the convergence of $\tilde{E}(k)$. Considering that $(20)$ is a linear difference equation in $\tilde{E}(k)$, it can be rewritten as

$$
\tilde{E}_{v}(k+1)=\tilde{A} \tilde{E}_{v}(k)+\tilde{W}
$$

where $\tilde{E}_{v}(k)$ and $\tilde{W}$ are the vector forms of $\tilde{E}(k)$ and $W$, respectively, $\tilde{A}$ is a constant matrix related to $A, L, C$ and $\tilde{\delta}$. Since $\tilde{E}$ is bounded for any bounded input $W$ and initial condition $\tilde{E}_{v}(0),(24)$ has bounded-input, bounded-output stability. This in turn implies that (24) has asymptotic stability. It follows that $\tilde{E}_{v}$ converges to a constant vector as $k \rightarrow \infty$ (because the input $\tilde{W}$ is constant). Therefore, $\tilde{E}=\lim _{k \rightarrow \infty} \tilde{E}(k)$ exists and is finite. Hence, (21) follows.

\subsection{Design of Estimator Gain}

We now discuss how to design $L$. From (21), it is natural to choose $L$ to minimize $\tilde{E}$. If $\delta$ (and thus $\tilde{\delta}$ ) is small and the Kalman gain $L_{K}$, which is the optimal $L$ when $\delta=0$, is not large, it is typically sufficient to choose $L=L_{K}$. In general, the following result can be used:

Theorem 3. The optimal $L$ that minimizes $\operatorname{Tr}(\tilde{E})$ in $(21)$ can be found by solving the following generalized discretetime algebraic Riccati equation for a symmetric and positive-definite matrix $\tilde{E}$ :

$$
\tilde{E}=A \tilde{E} A^{T}+B \Sigma_{w} B^{T}-\frac{A \tilde{E} C^{T} C \tilde{E} A^{T}}{\left(1+\tilde{\delta}^{2}\right)\left(C \tilde{E} C^{T}+\Sigma_{v}\right)}
$$

and the optimal estimator gain $L$ is given by

$$
L=\frac{1}{\left(1+\tilde{\delta}^{2}\right)\left(C \tilde{E} C^{T}+\Sigma_{v}\right)} A \tilde{E} C^{T}
$$

Equivalently, we can obtain $\tilde{E}$ in $(25)$ by solving the following convex optimization problem: $\min \operatorname{Tr}(Q)$, subject to: $\left[\begin{array}{ll}Q & I \\ I & P\end{array}\right] \geq 0$

$$
\left[\begin{array}{cccc}
P & P A & P B & \tilde{\delta} P A \\
A^{T} P & \left(1+\tilde{\delta}^{2}\right)\left(P+C^{T} \Sigma_{v}^{-1} C\right) & 0 & 0 \\
B^{T} P & 0 & \Sigma_{w}^{-1} & 0 \\
\tilde{\delta} A^{T} P & 0 & 0 & \left(1+\tilde{\delta}^{2}\right) P
\end{array}\right] \geq 0
$$

with the optimal $\tilde{E}$ given by $\tilde{E}=P^{-1}$.

Proof: Expanding the right-hand side of (21) and regrouping the terms, we get

$$
\begin{aligned}
\tilde{E}= & A \tilde{E} A^{T}+B \Sigma_{w} B^{T}-\frac{A \tilde{E} C^{T} C \tilde{E} A^{T}}{\left(1+\tilde{\delta}^{2}\right)\left(C \tilde{E} C^{T}+\Sigma_{v}\right)} \\
& +\left(L-A \tilde{E} C^{T} R^{-1}\right) R\left(L-A \tilde{E} C^{T} R^{-1}\right)^{T}
\end{aligned}
$$

where $R=\left(1+\tilde{\delta}^{2}\right)\left(C \tilde{E} C^{T}+\Sigma_{v}\right)$. Nullifying the term that involves $L$ will minimize $\tilde{E}$, which yields (25) and (26).

In order to show (27), consider the following modified version of (21):

$$
\begin{aligned}
\tilde{E}_{\Omega}= & (A-L C) \tilde{E}_{\Omega}(A-L C)^{T}+\tilde{\delta}^{2} L C \tilde{E}_{\Omega} C^{T} L^{T} \\
& +\left(1+\tilde{\delta}^{2}\right) L \Sigma_{v} L^{T}+B \Sigma_{w} B^{T}+\Omega
\end{aligned}
$$

where $\Omega=\Omega^{T} \geq 0$. It is clear that $\tilde{E}_{\Omega}$ is a monotonically increasing function of $\Omega$. Hence,

$$
\begin{aligned}
& \min _{\tilde{E}, L} \operatorname{Tr}(\tilde{E}), \quad \text { subject to: } \\
& \begin{aligned}
\tilde{E}>(A-L C) \tilde{E}(A-L C)^{T}+\tilde{\delta}^{2} L C \tilde{E} C^{T} L^{T} \\
\quad \\
\quad+\left(1+\tilde{\delta}^{2}\right) L \Sigma_{v} L^{T}+B \Sigma_{w} B^{T}
\end{aligned}
\end{aligned}
$$

gives the (unique) solution of $\tilde{E}$ to (21). Now rewriting the right-hand side of (30) as that of (28), we see that the optimal $L$ is given by (26) and the optimization problem above simplifies to

$$
\begin{aligned}
& \min _{\tilde{E}} \operatorname{Tr}(\tilde{E}), \quad \text { subject to: } \\
& \tilde{E}>A \tilde{E} A^{T}+B \Sigma_{w} B^{T}-\frac{A \tilde{E} C^{T} C \tilde{E} A^{T}}{\left(1+\tilde{\delta}^{2}\right)\left(C \tilde{E} C^{T}+\Sigma_{v}\right)} \\
& \quad=\frac{\tilde{\delta}^{2}}{1+\tilde{\delta}^{2}} A \tilde{E} A^{T}+B \Sigma_{w} B^{T} \\
& \quad+\left(1+\tilde{\delta}^{2}\right)^{-1} A\left(\tilde{E}^{-1}+C^{T} \Sigma_{v}^{-1} C\right)^{-1} A^{T} .
\end{aligned}
$$

Denoting $P=\tilde{E}^{-1}$ and pre- and post-multiplying the left and right sides of the above inequality by $P$ leads to:

$$
\begin{aligned}
P>\frac{\tilde{\delta}^{2}}{1+\tilde{\delta}^{2}} P A P^{-1} A^{T} P+P B \Sigma_{w} B^{T} P \\
\quad+\left(1+\tilde{\delta}^{2}\right)^{-1} P A\left(P+C^{T} \Sigma_{v}^{-1} C\right)^{-1} A^{T} P .
\end{aligned}
$$

Applying Schur's complement, the latter inequality becomes the matrix inequality in (27). Finally, it is easy to check that $\min \operatorname{Tr}(\tilde{E})$ is the same as

$$
\min \operatorname{Tr}(Q), \text { subject to }\left[\begin{array}{ll}
Q & I \\
I & P
\end{array}\right] \geq 0
$$

because the latter reaches the optimum when $Q=P^{-1}=\tilde{E}$. 


\subsection{Illustrative Example}

We now give an example to demonstrate the accuracy of the estimate $\tilde{E}$ and the proposed estimator gain design. We will call the optimal $L$ in (26) a robust estimation gain due to the fact that it is designed to mitigate quantization errors. The gain $L$ designed without considering quantization errors will be referred to as the Kalman gain.

The example we consider is a low-pass filtered random process corrupted by a measurement noise. More specifically, the system model of the filter is given by (1) with

$$
\begin{gathered}
A=\left[\begin{array}{ccccc}
2.4744 & -2.8110 & 1.7038 & -0.5444 & 0.0723 \\
1 & 0 & 0 & 0 & 0 \\
0 & 1 & 0 & 0 & 0 \\
0 & 0 & 1 & 0 & 0 \\
0 & 0 & 0 & 1 & 0
\end{array}\right] \\
B^{T}=\left[\begin{array}{lllll}
1 & 0 & 0 & 0 & 0
\end{array}\right] \\
C=\left[\begin{array}{lllll}
0.245 & 0.236 & 0.384 & 0.146 & 0.035
\end{array}\right]
\end{gathered}
$$

and $\Sigma_{w}=1$. Different values of $\Sigma_{v}$ will be considered. The filter has a normalized bandwidth of approximately 0.25 (where 1 corresponds to the Nyquist bandwidth).

Two cases, $\Sigma_{v}=1$ and $\Sigma_{v}=1 / 16$, are tested. The range of $\delta$ for the tests is chosen to be $\left[\begin{array}{ll}0 & 0.3\end{array}\right]$. For a given $\Sigma_{v}$ and $\delta$, we have designed two estimator gains, one taken as the Kalman gain designed by ignoring the quantization error and the other being the robust gain computed using (26). Quadratic stability of (9) is verified using (17) for both gains at $\delta=0.3$.

Figures 3 and 4 show the simulated values of $\operatorname{Tr}(E)$ for both estimator gains along with their estimates $\operatorname{Tr}(\tilde{E})$. Figure 3 is for $\Sigma_{v}=1$ and Figure 4 for $\Sigma_{v}=1 / 16$. From these figures, we see that when the measurement noise is relatively large $\left(\Sigma_{v}=1\right)$, the Kalman gain performs well (and is actually slightly better than the robust gain). But when the measurement noise is relatively low $\left(\Sigma_{v}=1 / 16\right)$, the robust gain performs significantly better than the Kalman gain. This is because when $\Sigma_{v}$ is small, Kalman estimation relies heavily on the measurement, which is thus sensitive to quantization errors. In contrast, the robust gain is designed to cope with quantization errors, so it performs better when $\Sigma_{v}$ is small and the quantization error dominates.

Also seen in Figures 3 and 4 is that, in all cases, the estimate $\operatorname{Tr}(\tilde{E})$ matches the actual $\operatorname{Tr}(E)$ very well, especially for small $\delta$. Since we typically want the quantizer not to introduce too much additional estimation error, a relatively small $\delta$ needs to be used. This example shows that the estimate $\tilde{E}$ is reasonably accurate.

\section{STATE ESTIMATION WITH FINITE-LEVEL QUANTIZATION}

A finite-level quantizer can be designed by simply truncating a logarithmic quantizer. We define a $2 N$-level logarithmic quantizer with $0<\rho<1$ as

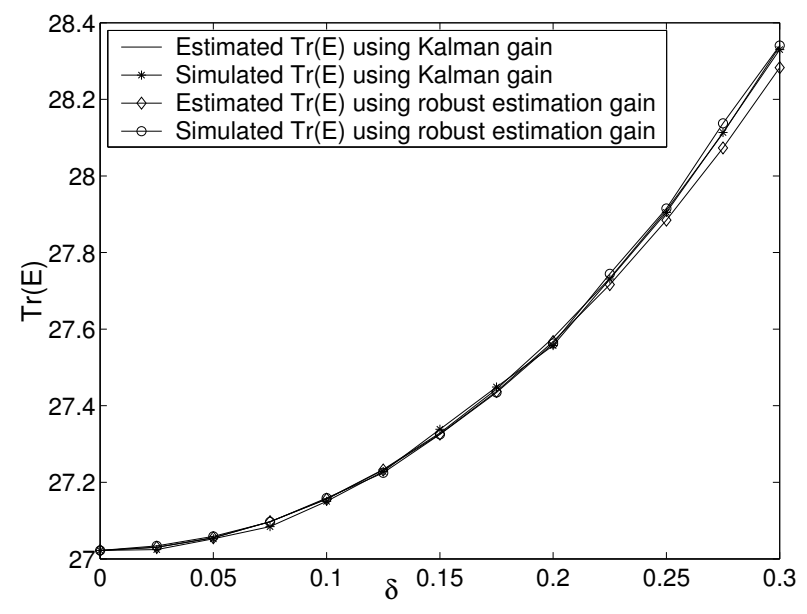

Fig. 3. Logarithmic quantization for $\Sigma_{v}=1$.

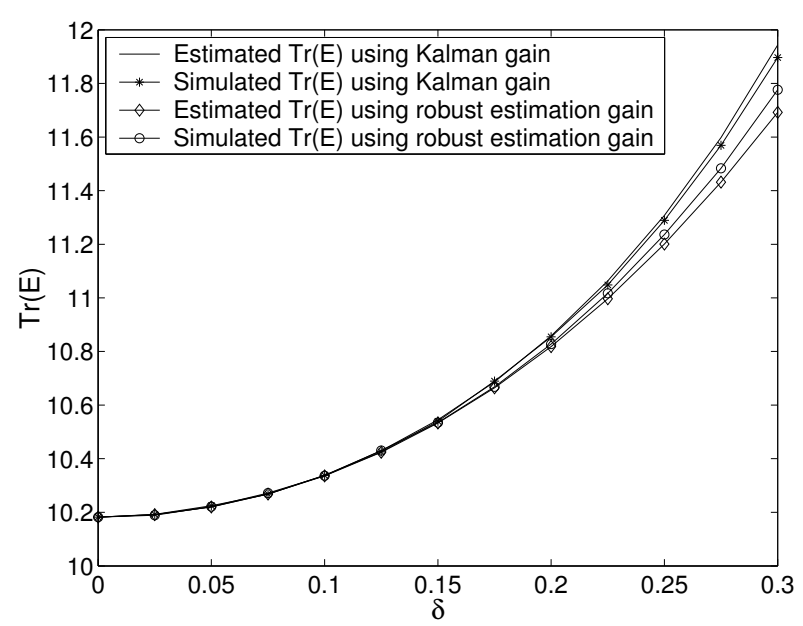

Fig. 4. Logarithmic quantization for $\Sigma_{v}=1 / 16$.

$$
Q(y)= \begin{cases}\rho^{i} \mu_{0}, & \text { if } \frac{1}{1+\delta} \rho^{i} \mu_{0}<y \leq \frac{1}{1-\delta} \rho^{i} \mu_{0}, \\ & 0<i<N-1 \\ \rho^{N-1} \mu_{0}, & \text { if } 0 \leq y \leq \frac{1}{1-\delta} \rho^{N-1} \mu_{0} \\ \mu_{0}, & \text { if } y>\mu_{0} /(1+\delta) \\ -Q(-y), & \text { if } y<0 .\end{cases}
$$

Recall that in the infinite-level quantization case $\mu_{0}$ does not affect $\varepsilon_{q}(k)$ much. In the finite-level quantization case, the parameter $\mu_{0}$ needs to be optimized. Also, in the infinite-level case, a smaller value of $\delta$ leads to a smaller quantization error. But this is no longer true in the finitelevel case because a smaller $\delta$ for a fixed $N$ means that the input range for the quantizer without quantization is smaller. Therefore, both $\mu_{0}$ and $\delta$ need to be optimized for a given number of quantization levels.

To set up this optimization problem, we assume that Conditions $\mathrm{C} 1$ and $\mathrm{C} 2$ hold. Recall that the zero-mean property is guaranteed when $x(0)-\bar{x}_{0}, w(k)$ and $v(k)$ have even probability density functions. The optimization problem can be written as follows:

$$
\min J\left(\mu_{0}, \delta\right)
$$


where $J\left(\mu_{0}, \delta\right)$ is the variance of the quantization error. It turns out that the optimal $J\left(\mu_{0}, \delta\right)$ depends on $\mu=\mu_{0} / \sigma_{\varepsilon}$ rather than $\mu_{0}$ and $J\left(\mu_{0}, \delta\right)$ linear in $\sigma_{\varepsilon}$, i.e., we may write

$$
J\left(\mu_{0}, \delta\right)=\tilde{J}(\mu, \delta) \sigma_{\varepsilon} .
$$

For the given $N, \tilde{J}(\mu, \delta)$ can be numerically optimized and the result is shown in Table 1 . In the table, $N_{b}$ denotes the number of quantization bits, namely $2^{N_{b}}=2 N$.

\begin{tabular}{|c|c|c|c|c|c|}
\hline$N_{b}$ & $N$ & $\delta$ & $\rho$ & $\mu_{0} / \sigma_{\varepsilon}$ & $J\left(\mu_{0}, \delta\right) / \sigma_{\varepsilon}$ \\
\hline 2 & 2 & 0.5338 & 0.3040 & 1.7699 & 0.1457 \\
\hline 3 & 4 & 0.3253 & 0.5091 & 2.7220 & 0.04892 \\
\hline 4 & 8 & 0.1909 & 0.6794 & 3.4887 & 0.01568 \\
\hline 5 & 16 & 0.1095 & 0.8026 & 4.0931 & 0.00494 \\
\hline 6 & 32 & 0.0619 & 0.8834 & 4.5774 & 0.00153 \\
\hline 7 & 64 & 0.0346 & 0.9331 & 4.9779 & 0.00047 \\
\hline 8 & 128 & 0.0191 & 0.9625 & 5.3134 & 0.00014 \\
\hline
\end{tabular}

Table 1. Optimized quantization density.

Using the optimized $\tilde{J}(\mu, \delta)$, we can revise $(21)$ to

$$
\begin{aligned}
\tilde{E}= & (A-L C) \tilde{E}(A-L C)^{T}+B \Sigma_{w} B^{T} \\
& +L \Sigma_{v} L^{T}+\tilde{J}(\mu, \delta) L\left(C \tilde{E} C^{T}+\Sigma_{v}\right) L^{T} .
\end{aligned}
$$

\subsection{Illustrative Example}

The results above are demonstrated using the same example as in the previous section. The simulations are shown in Figures 5 and 6 , again for $\Sigma_{v}=1$ and $\Sigma_{v}=1 / 16$, respectively. Three observations are made. Firstly, with about $4 \sim 5$ bits of quantization, the quantized estimator has its estimation error variance only marginally larger than in the case without quantization. Secondly, the improvement by the robust estimation gain is marginal when $N_{b} \geq 4$, but more noticeable when $N_{b}$ is small, especially when the measurement noise is relatively small. For the case when $\Sigma_{v}=1 / 16$ and $N_{b}=2$, the Kalman gain yields $\operatorname{Tr}(E) \approx 56$. If we decrease $\Sigma_{v}$ further, the Kalman gain will yield an unstable estimator. Thirdly, our estimate for the estimation error is very accurate (with less than $0.1 \%$ relative error for $N_{b} \geq 3$ ).

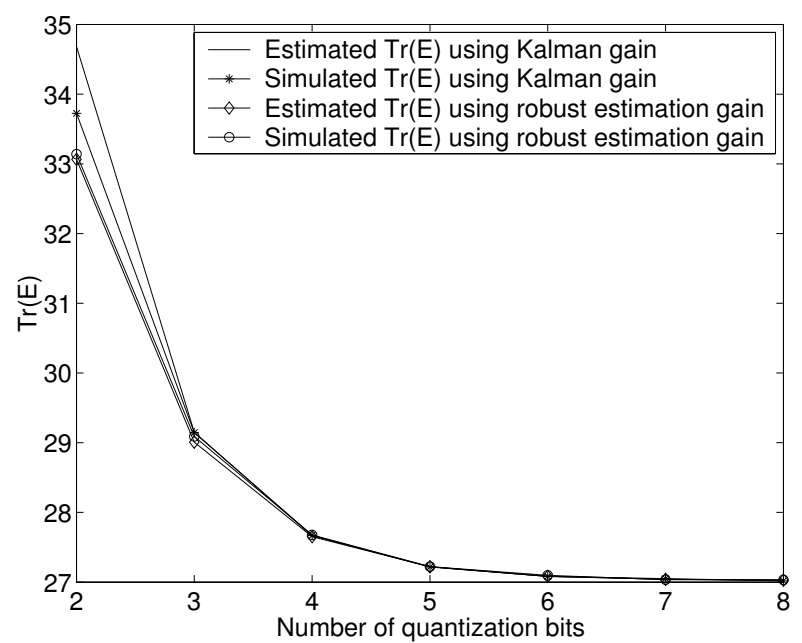

Fig. 5. Finite-level quantization for $\Sigma_{v}=1$.

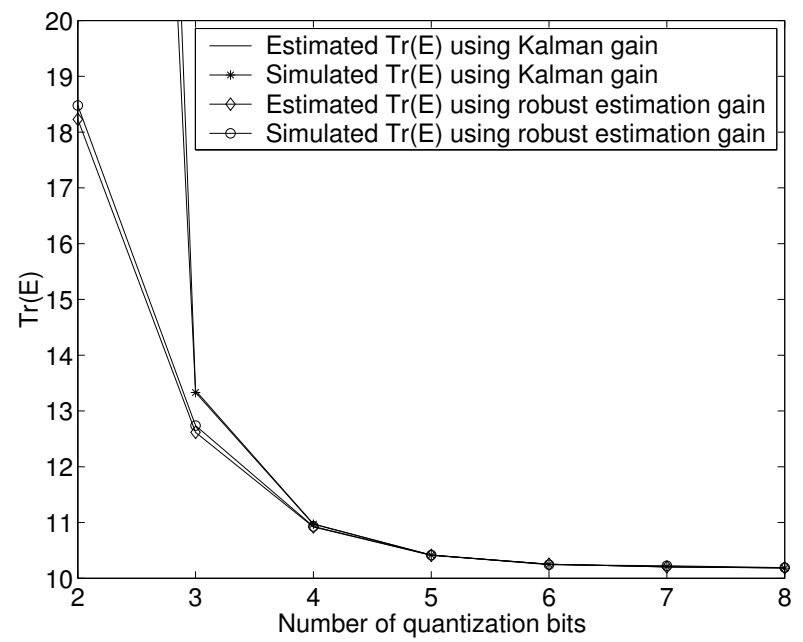

Fig. 6. Finite-level quantization for $\Sigma_{v}=1 / 16$.

\section{ACKNOWLEDGEMENTS}

This work was supported in part by "Conselho Nacional de Desenvolvimento Científico e Tecnológico - CNPq", Brazil, under grants 30.2317/02-3/PQ and 45.4565/06-2/APV.

\section{REFERENCES}

W. S. Wong and R. W. Brockett, "Systems with finite communication bandwidth constraints II: Stabilization with limited information feedback," IEEE Trans. Automat. Contr., vol. 44, pp. 1049-1053, 1999.

J. Baillieul, "Feedback designs in information-based control," Proc. Stochastic Theory and Control Workshop, Kansis, pp. 35-57, 2001.

R. W. Brockett and D. Liberzon, "Quantized feedback stabilization of linear systems," IEEE Trans. Automat. Contr., vol. 45, pp. 1279-1289, 2000.

N. Elia, "Design of hybrid systems with guaranteed performance," Proc. 39th IEEE Conf. Decision Control, pp. 993-998, Sydney, Australia, Dec. 2000.

N. Elia and K. Mitter, "Stabilization of linear systems with limited information," IEEE Trans. Automat. Contr., vol. 46, pp. 1384-1400, 2001.

G. N. Nair and R. J. Evans, "Exponential stabilization of multi-dimensional linear systems," Automatica, vol. 39, pp. 585-593, 2003.

S. Tatikonda and S. Mitter, "Control under communication constraints," IEEE Trans. Automat. Contr., vol. 49, pp. 1056-1068, 2004.

M. Fu and L. Xie, "The sector bound approach to quantized feedback control," IEEE Trans. Automat. Contr., vol. 50, pp. 1698-1711, 2005.

M. Fu and L. Xie, "Finite-level quantized feedback control for linear systems," Proc. 45th IEEE Conf. Decision Control, pp. 1117-1122, San Diego, CA, Dec. 2006.

A. Packard and J. C. Doyle, "Quadratic stability with real and complex perturbations," IEEE Trans. Automat. Contr., vol. 35, pp. 198-201, 1990.

B. D. O. Anderson and J.B. Moore, Optimal Filtering, Prentice-Hall, Englewood Cliffs, NJ, 1979.

C. E. de Souza and L. Xie, "On the discrete-time bounded real lemma with application in the characterization of static state-feedback $H_{\infty}$ controller," Systems \& Control Letts., vol. 18, pp. 61-71, 1992. 